\title{
Fortbildung, Hands-on und eine Überraschungstorte: Der 9. RadiologieKongressRuhr in Bildern
}

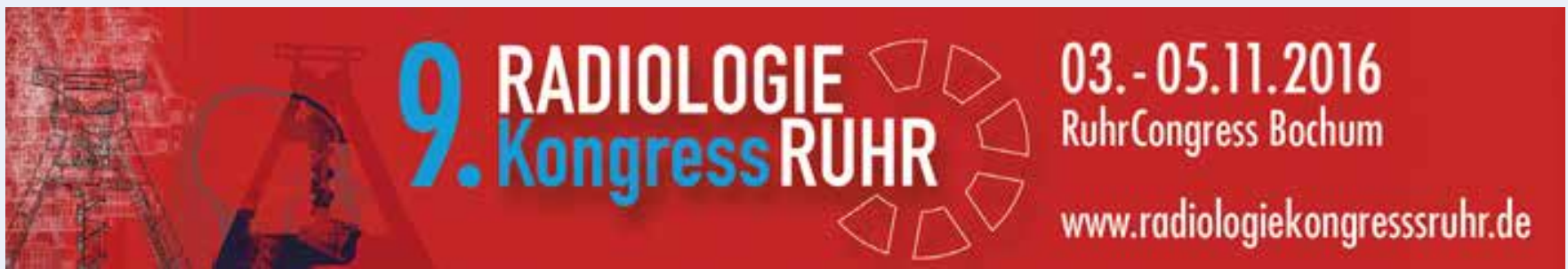

\section{Der RKR - Eine Erfolgs- geschichte}

Längst ist der RadiologieKongressRuhr in der Fortbildungswelt der Radiologie angekommen und hat sich in den letzten Jahren zu einem wichtigen Termin für Radiologen, MTRA und Industrievertreter entwickelt. Nach dem Deutschen Röntgenkongress ist er der zweitgrößte Kongress der medizinischen Bildgebung in Deutschland. Ein Umstand, der stolz macht, aber auch an-

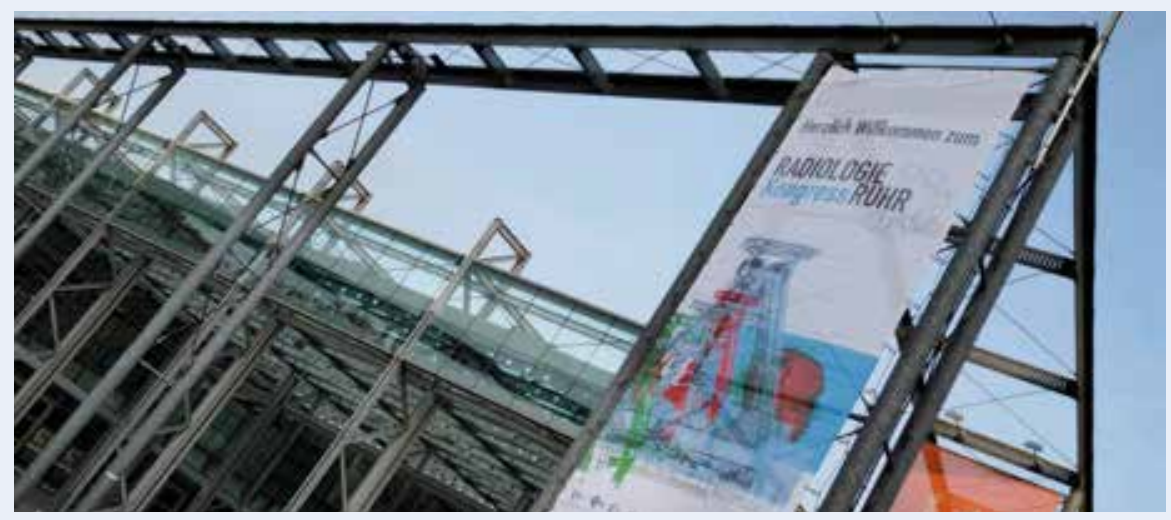


treibt, immer besser zu werden und den wachsenden Anforderungen der Teilnehmenden gerecht zu werden. Dies ist auf dem 9. RadiologieKongressRuhr gelungen! Die Zahl der Teilnehmenden ist wieder gestiegen ebenso wie das Angebot an Fortbil-

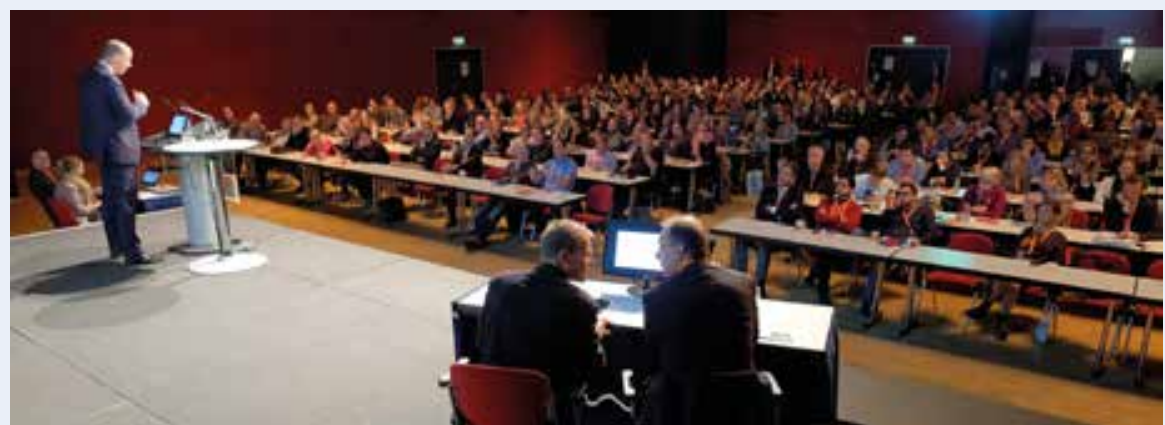

dungsveranstaltungen. 1370 Radiologen, MTRA, MTRA-Schüler, Studierende und Industrievertreter fanden Anfang November ihren Weg in den RuhrCongress nach Bochum.

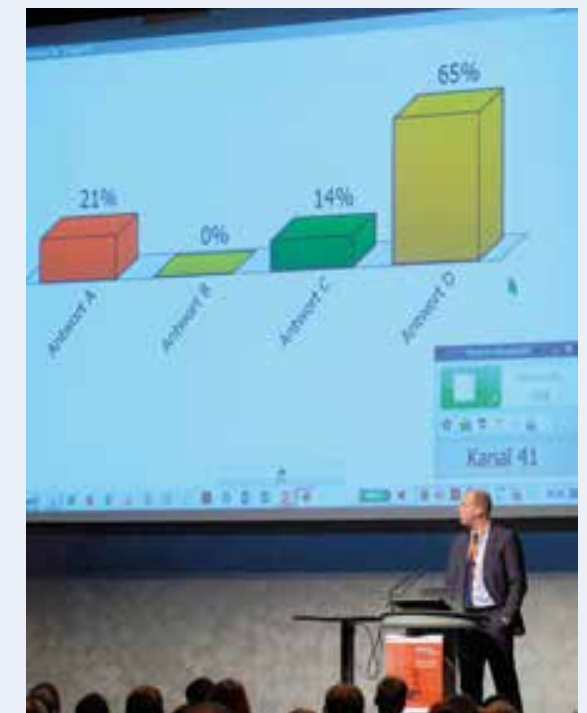

\section{Hands-on-Workshops}

Ein unbestrittener Höhepunkt waren die Hands-on-Workshops, die zu den Themen „Stroke“ und „Becken / Bein“ angeboten wurden. Gemeinsam mit der engagierten Unterstützung zahlreicher Firmen konnten wir dieses attraktive Angebot für die Teilnehmenden weiter ausbauen und werden dies aufgrund der durchweg positiven Resonanz auch im kommenden Jahr weiter tun.
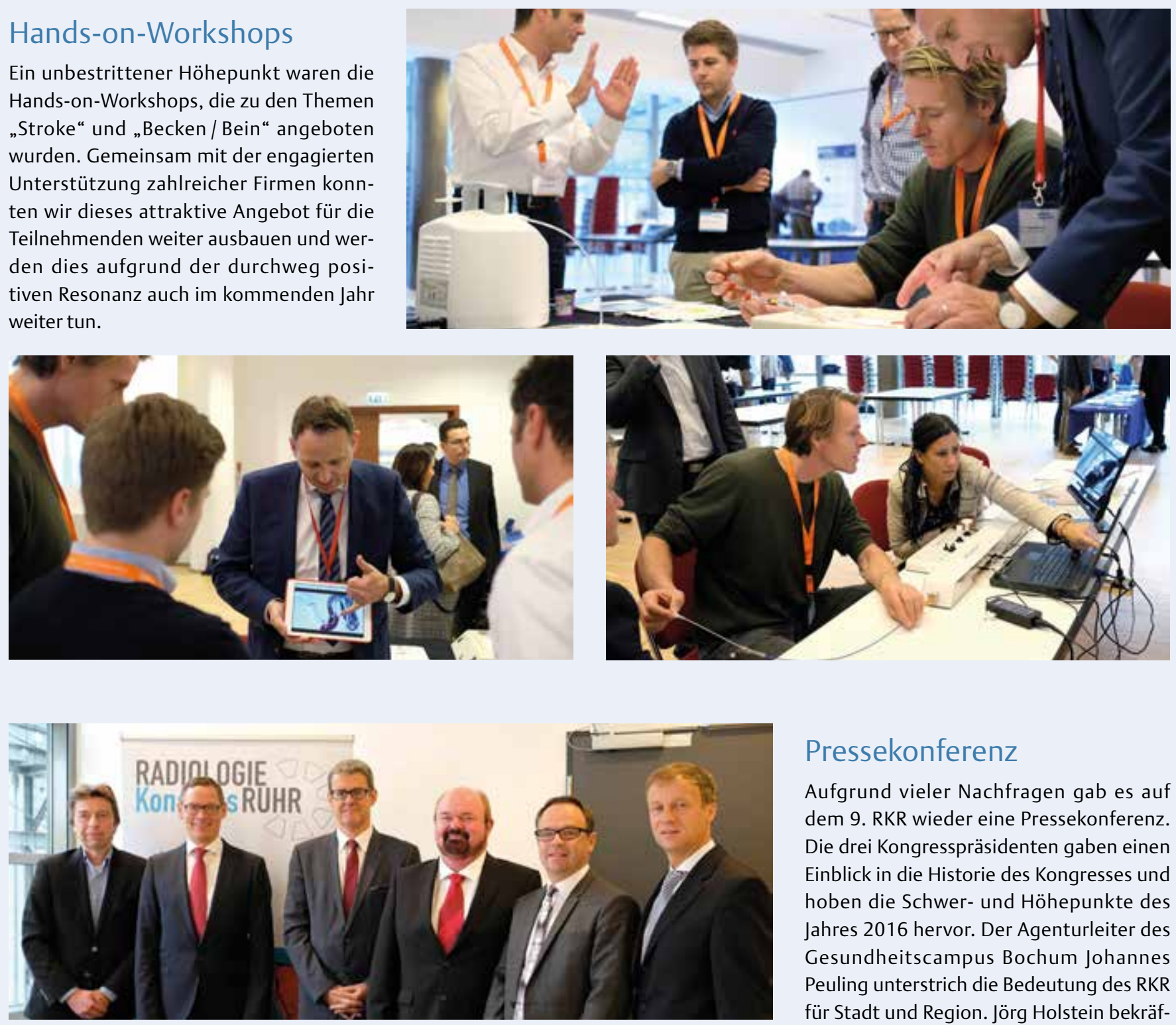

Pressekonferenz

Aufgrund vieler Nachfragen gab es auf dem 9. RKR wieder eine Pressekonferenz. Die drei Kongresspräsidenten gaben einen Einblick in die Historie des Kongresses und hoben die Schwer- und Höhepunkte des Jahres 2016 hervor. Der Agenturleiter des Gesundheitscampus Bochum Johannes Peuling unterstrich die Bedeutung des RKR für Stadt und Region. Jörg Holstein bekräf- 
tige als Geschäftsführer des langjährigen Hauptsponsors VISUS die hervorragende Zusammenarbeit und den gegenseitigen Nutzen von Industrie und RKR.

\section{Der RKR und die Stadt Bochum}

Die Stadt Bochum und der RKR sind seit Beginn an eng miteinander verbunden. Daher freut es uns sehr, dass der Oberbürgermeister der Stadt Bochum Herr Thomas

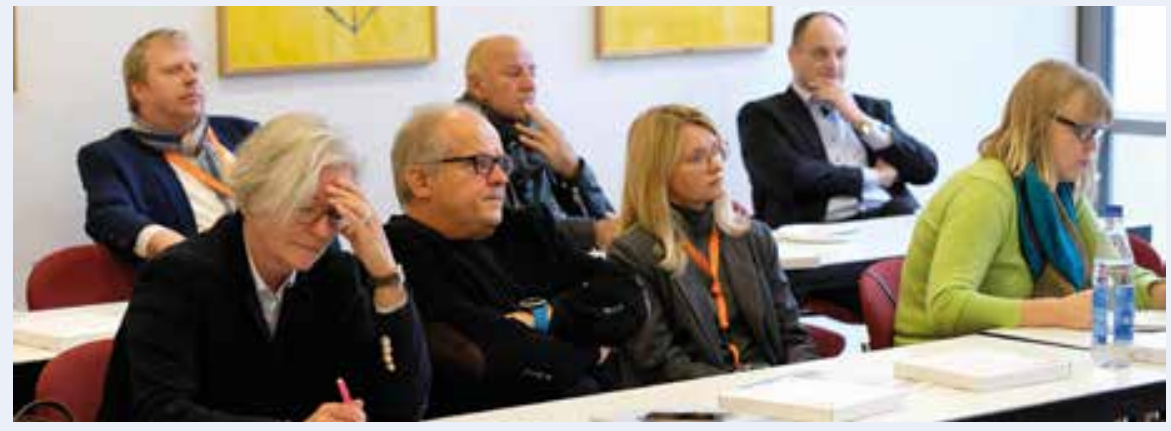

Eiskirch den traditionellen Empfang der Stadt am ersten Kongressabend feierlich eröffnete. Die große Industrieausstellung mit zahlreichen lokalen Firmen wollte er sich auch nicht entgehen lassen und sprach am kommenden Morgen gemeinsam mit den Kongresspräsidenten mit teilnehmenden Firmen über ihre neuesten Ent- wicklungen. Glücklicherweise erfuhren wir am Vorabend, dass Herr Eiskirch am Kongressfreitag seinen Geburtstag feiert und konnten ihn mit einer Torte und einem Glas Sekt überraschen.
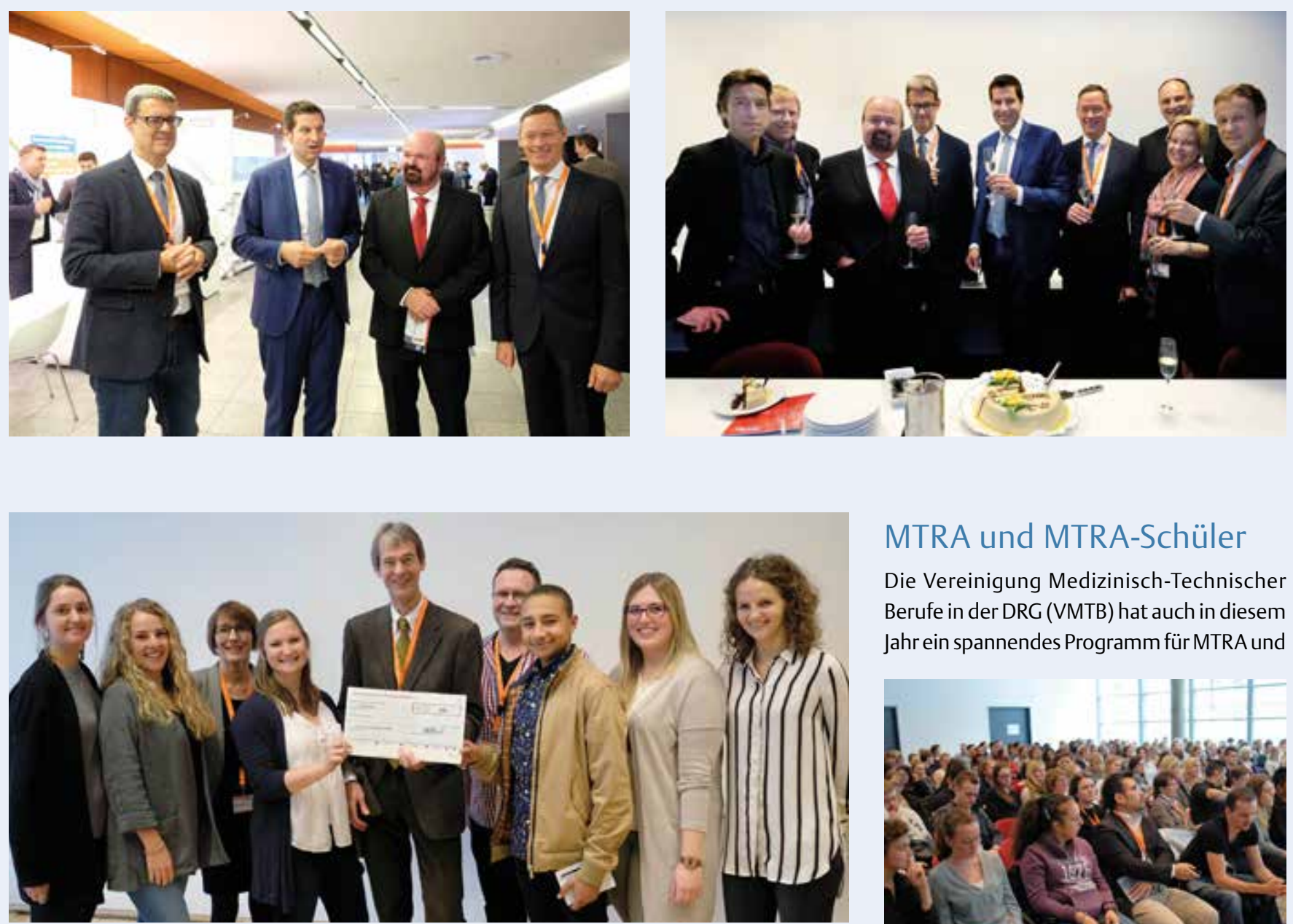

\section{MTRA und MTRA-Schüler}

Die Vereinigung Medizinisch-Technischer Berufe in der DRG (VMTB) hat auch in diesem Jahr ein spannendes Programm für MTRA und

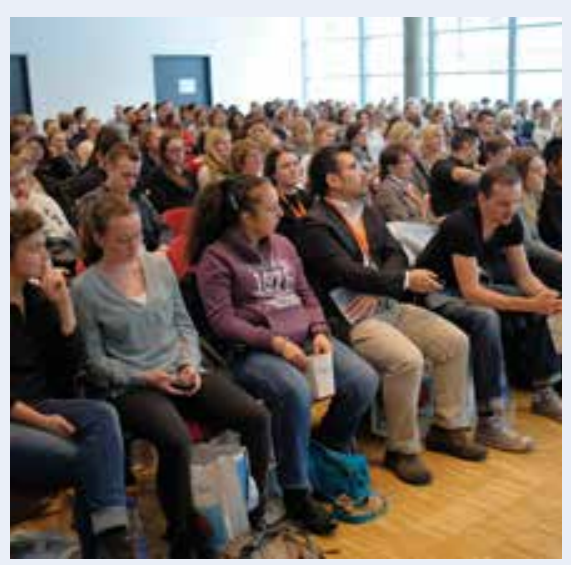




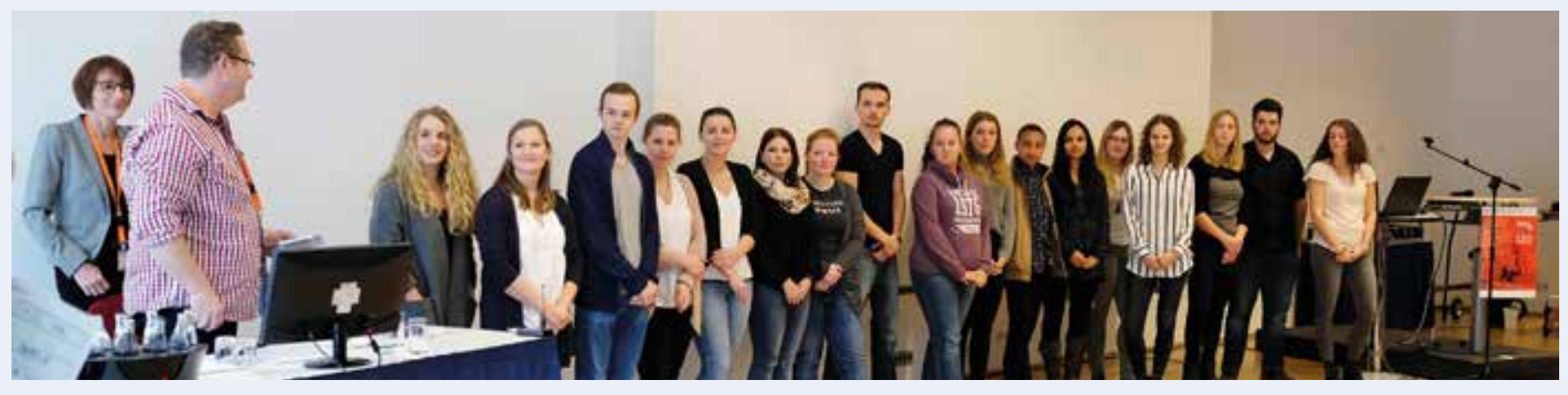

MTRA-Schüler zusammengestellt. Am Kongressfreitag kamen 175 MTRA zum ganztätigen Programm, ein deutlicher Zuwachs im Vergleich der letzten Jahre. Der seit Jahren beliebte MTRA-Schüler-Donnerstag brachte den Saal erneut an seine Kapazitätsgrenze, nicht zuletzt wegen der hochkarätigen Vorträge, die sechs Schulen Ihren Mitschülern präsentierten. Am Ende gab es sogar erstmals einen Preis für die beste vortragende Schule, den sich die Schulen aus Münster und Köln teilten: 500 Euro, gestiftet von der RWRG. Alle anderen Schulen erhielten Gutscheine.

\section{Radiologischer Nachwuchs}

Schon im dritten Jahr bietet der RKR nun auch den Radiologinnen und Radiologen von morgen eine eigene Plattform. Im „Programm für den radiologischen Nachwuchs“ ging es in diesem Jahr um die Bildgebung des Abdomen. Ein voller Saal bestätigt uns hier auf dem richtigen Weg zu sein und für die Nachwuchsarbeit in der Radiologie einen wichtigen Teil beizutragen.
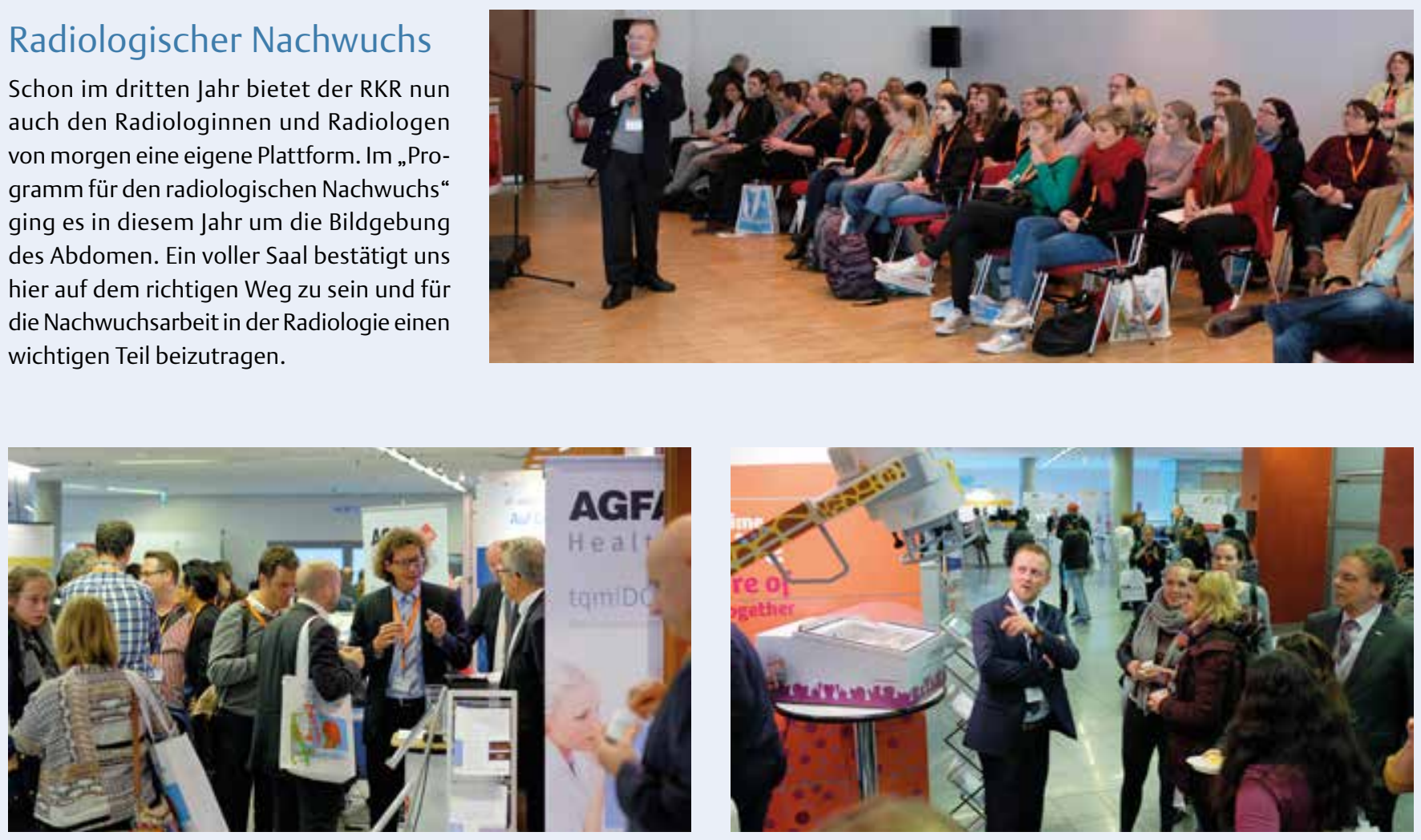

\section{Industrie}

Die Industrie ist ein unverzichtbarer Bestandteil des RKR. Seit jeher besteht hier eine enge Verbindung und dieses gute Verhältnis ist über die Jahre immer stabiler geworden. Da die Industriefläche eng eingebunden ist in den Kongress - kaum ist man aus dem Vortragsraum raus, steht man schon mitten drin - kommen zahlreiche Firmen jedes Jahr wieder gerne auf den RKR. Manche sogar schon seit den Anfängen

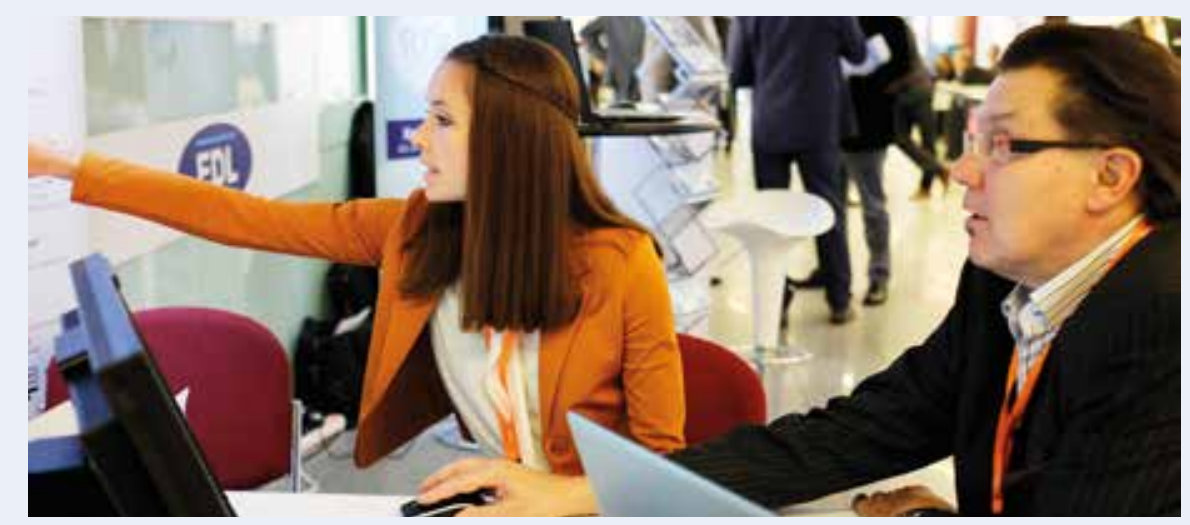


2008. Insgesamt 61 Firmen aus dem RheinRuhr-Gebiet und ganz Deutschland haben auf 466 qm Industriefläche ihre medizintechnischen Neuerungen präsentiert und kamen mit den Besuchern - bei Kaffee, Eis und Popcorn - ins Gespräch.
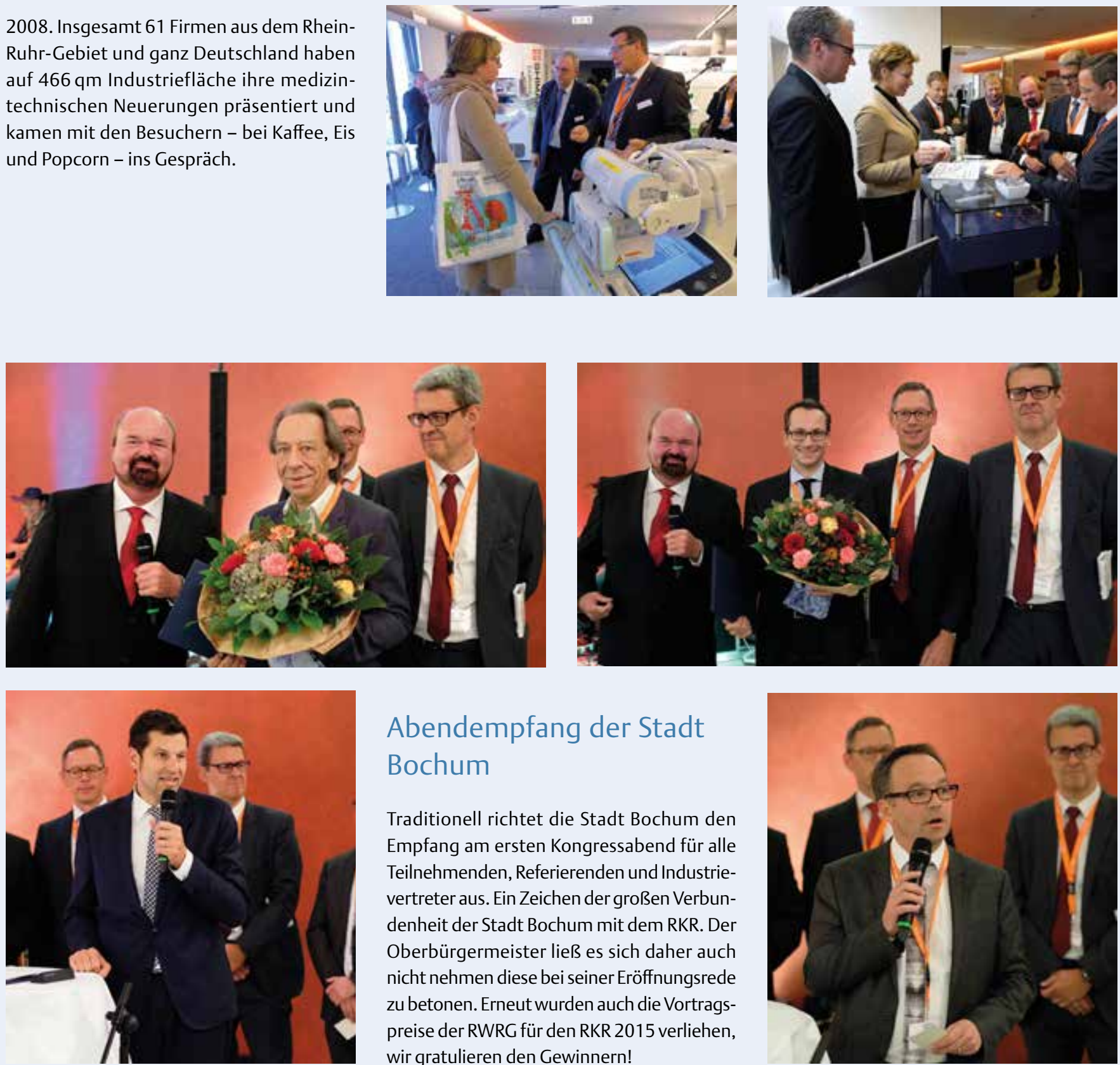

Abendempfang der Stadt Bochum

Traditionell richtet die Stadt Bochum den Empfang am ersten Kongressabend für alle Teilnehmenden, Referierenden und Industrievertreter aus. Ein Zeichen der großen Verbundenheit der Stadt Bochum mit dem RKR. Der Oberbürgermeister ließ es sich daher auch nicht nehmen diese bei seiner Eröffnungsrede zu betonen. Erneut wurden auch die Vortragspreise der RWRG für den RKR 2015 verliehen, wir gratulieren den Gewinnern!
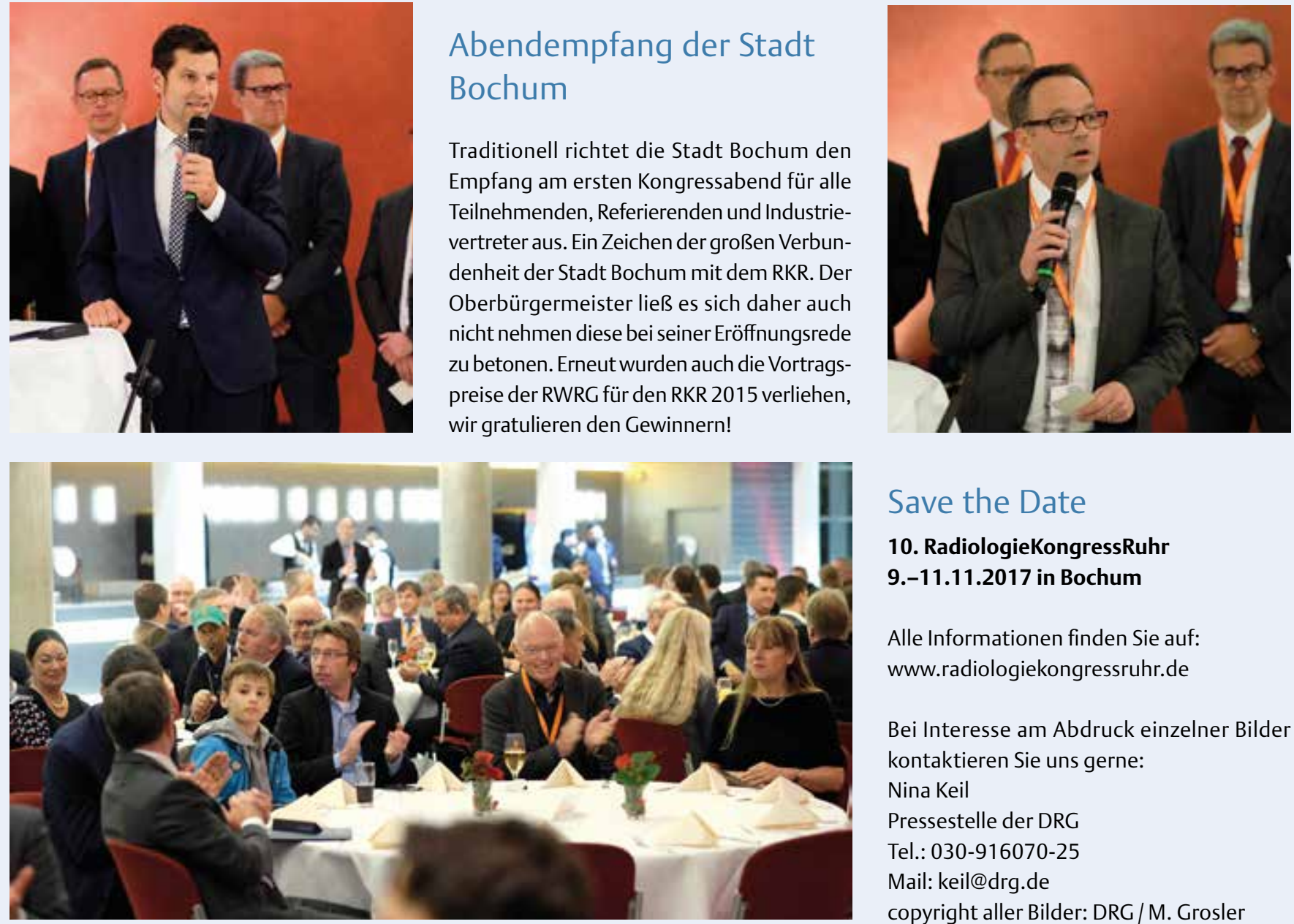

Save the Date

\section{RadiologieKongressRuhr} 9.-11.11.2017 in Bochum

Alle Informationen finden Sie auf: www.radiologiekongressruhr.de

Bei Interesse am Abdruck einzelner Bilder kontaktieren Sie uns gerne:

Nina Keil

Pressestelle der DRG

Tel.: 030-916070-25

Mail: keil@drg.de

copyright aller Bilder: DRG / M. Grosler 